\title{
A Mathematical Model of the Skype VoIP Congestion Control Algorithm
}

\author{
Luca De Cicco, Saverio Mascolo and Vittorio Palmisano
}

\begin{abstract}
The Internet is changing from being only an efficient platform for data delivery to become also a platform for audio/video applications. The stability of the traditional Internet is due to the TCP congestion control algorithm. However, the TCP congestion control is not optimal for VoIP applications because of its retransmission mechanism and additive increase multiplicative decrease sliding window control. As a consequence, VoIP applications often employ proprietary and hidden congestion control algorithms executed over the UDP protocol. In this paper we focus on Skype audio, which is the most known and used VoIP application, in order to derive a mathematical model of its congestion control algorithm and evaluate properties such as efficiency in network utilization while avoiding congestion, which is particularly detrimental for VoIP traffic. To the purpose, the controller input/output variables are first identified and then their dynamic relation is described in the form of a hybrid automaton. Main findings are: (1) Skype does not implement a delay based control; (2) the loss ratio is the main input that affects the sending rate; (3) the sending rate matches the available bandwidth with a finite error.
\end{abstract}

\section{INTRODUCTION AND RELATED WORK}

It is well known that the main driver of the Internet stability is the TCP congestion control algorithm developed by V. Jacobson for data delivery [1]. Nowadays, the efficient transport of multimedia flows is becoming a hot issue due to the booming of applications based on multimedia content delivery. However, TCP is not well suited for audio/video delivery due to its retransmission mechanism and sliding window control. As a consequence, audio and video applications are in practice transported by hidden proprietary protocols using the UDP protocol.

In this area Voice over IP plays a key role as it is shown by the success of Skype application for end users and by the large deployment of SIP-based networks. This explosive growth poses challenges to telecom operators and ISPs both from the point of view of business model and network stability. In spite of this explosive growth it is not yet clear what will be the impact of multimedia traffic on the stability of the Internet when a very large amount of this traffic will populate the network.

This work was partially supported by Financial Tradeware PLC

Luca De Cicco is a PhD student of Dipartimento di Elettrotecnica ed Elettronica, Politecnico di Bari, Via Orabona 4, Italy ldecicco@poliba.it

Saverio Mascolo is a Faculty member of Dipartimento di Elettrotecnica ed Elettronica, Politecnico di Bari, Via Orabona 4, Italy mascolo@poliba.it

Vittorio Palmisano is a $\mathrm{PhD}$ student in Dipartimento di Elettrotecnica ed Elettronica, Politecnico di Bari, Via Orabona 4, Italy vpalmisano@poliba.it
To this purpose, mathematical models play a major role in understanding the fundamental properties such as the stability of large scale and complex communication systems [2]. Up to now the only congestion control algorithm for data networks that has been modelled is the standard TCP congestion control and its variants [3],[4],[5],[6]. This is due to the fact that the proposed TCP congestion control algorithms are fully disclosed and well described in scientific literature and in standardization bodies such as the IETF.

The only standardization effort proposed within the IETF as a possible congestion control algorithm for multimedia flows is represented by the TCP Friendly Rate Control (TFRC) protocol [7], [8]. In spite of this effort, as a matter of fact, all commercial audio/video applications run over UDP and we conjecture that they implement some congestion control algorithm at the application level.

One of the most important Voice over IP (VoIP) applications is Skype, with an ever growing user-base which today counts more than 10 million concurrent users.

Skype is a closed source application, which employs a proprietary protocol that is hidden by using AES encryption [9]. Some efforts have been spent so far to investigate the features of the protocol by using reverse-engineering techniques [9].

In this work we do no not address the Skype signalling protocol (call establishment, call teardown) already investigated in [10], whereas we focus on the dynamics of the sending rate produced by Skype. In [11], [12] we have shown that Skype reacts to network congestion by reducing the sending rate, thus being able to match the link capacity to some extent.

In this work we propose a mathematical model of how Skype VoIP tracks the network available bandwidth in the presence of variable network conditions. The issue is complex for several reasons: (1) the protocol behaviour is hidden by AES encryption; (2) the input variables that drive the controller are unknown; (3) it is very much reasonable to conjecture that the controller implements a complex switching dynamics due to the use of if-the-else decisional blocks.

We will start by considering Skype as it is: a pure black box of which we do not know the controller inputs and we are not able to inspect the feedback packets because they are encrypted.

In order to carry out the investigation, we have set up a local testbed where different network parameters such as link delays and capacities, loss rates and queue sizes can be varied. All those variables are candidate for being inputs to the controller. In particular, to identify the controller inputs 


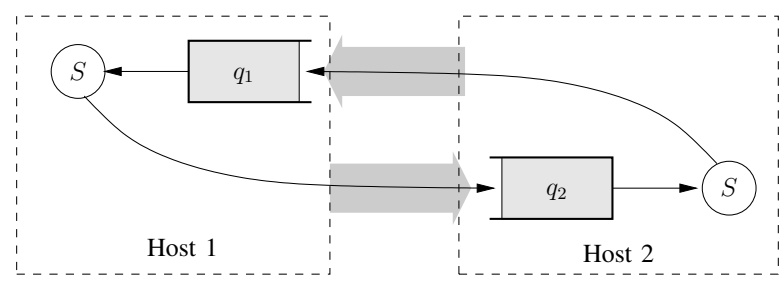

Fig. 1. Experimental testbed employed in the investigation

we have designed a proper set of experiments to evaluate the response of Skype to any input. Of course, we expect that some of these inputs will have stronger effects on the output of the controller (like the available bandwidth and the packet loss rate) whereas others will have weaker ones. In particular, we identify the controller inputs and outputs and we propose a hybrid automaton to describe the controller switching dynamics. At our best knowledge this is the first attempt to derive a mathematical model of the congestion control algorithm used in VoIP applications such as Skype.

The rest of the paper is organized as follows: in Section II we describe the experimental testbed that has been set up in order to investigate the Skype congestion controller; in Section III we show the effects of the candidate inputs on the output of the controller; in Section IV we present a mathematical model of Skype sending rate when congestion occurs and we derive a hybrid switching dynamic model of a Skype flow when accessing a bottleneck; a stability analysis is also carried out. Finally Section V concludes the paper and outlines further research work.

\section{EXPERIMENTAL TESTBED}

In order to investigate how Skype adapts to variations in the available bandwidth we have set up a local testbed using a measurement tool we have developed. We have routed all packets generated from Skype application to the ingress queues $q_{1}$ and $q_{2}$ of each host as it is shown in Figure 1 . The measurement tool allows delays, available bandwidth and buffer size of each queue be set by the user. It is worth noticing that the described set-up implements an emulated environment similar to that of Dummynet [13], the only difference being that in our case we can use two hosts instead of three.

On each host we have installed Skype $(S)$ and we have collected logfiles by tracing the per-flow data arriving to and departing from the queue. By comparing data at the input of the queue and at its output, we have been able to compute packet drop rates and goodputs for Skype flows. Goodput, throughput and loss rate are defined as follows: goodput $=$ $(\Delta s-\Delta l) / \Delta T$, throughput $=\Delta s / \Delta T$, and loss rate $=$ $\Delta l / \Delta T$, where $\Delta s$ is the number of bits sent in the period $\Delta T, \Delta l$ is the number of bits lost in the same period. We have considered $\Delta T=0.4 \mathrm{~s}$ in our measurements.

Our testbed is able to log jitter, RTT, packet loss ratio as declared by the Skype application in the "Technical Call Infos" tooltip.

Finally, it is worth noticing that Skype flows are generated

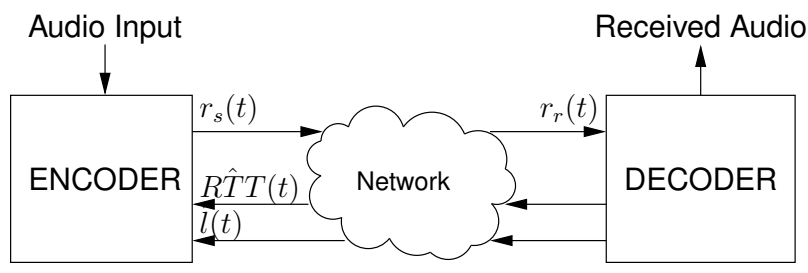

Fig. 2. Schematic of a Skype audio call over the Internet

using always the same audio sequence by hijacking audio $\mathrm{I} / \mathrm{O}^{1}$ to enforce experiments reproducibility. From now on, the $R T T$ of the connection is set at $100 \mathrm{~ms}$ and the queue size is set equal to the bandwidth delay product unless otherwise specified.

\section{EXPERIMENTS TO INVESTIGATE THE SKYPE CONGESTION CONTROL}

As we have already mentioned in the introduction, modeling the adaptation algorithm employed by Skype to match network available bandwidth is made complex by the fact that the source code of the application is not available and the application uses a proprietary and undisclosed communication protocol which is hidden by means of AES encryption.

In this investigation we consider the Skype sending rate as the output of the controller dynamics we are trying to model. At first we will determine what are the inputs and then how do they affect the output. We will consider as possible inputs to the controller the following variables: i) the end-to-end round trip time (RTT) experienced by the connection; ii) the packet loss rate. It is worth to notice that, since those two variables can be easily measured end-to-end, they are often employed in congestion control algorithms as feedback signals to detect network congestion.

For this reason congestion control algorithms are often classified as follows: i) delay-based algorithms, which infer congestion by monitoring either the one way delay, the end-to-end round trip time (RTT) or the queueing delay (examples of congestion control belonging to this category are Fast and Vegas TCP); ii) loss-based algorithms, which infer congestion based on packet loss events such as in the case of TCP NewReno, which is the congestion control standardized by IETF and its variants; iii) mixed loss and delay based algorithms, which use both delay and packet losses as feedbacks such as in the case of recently proposed TCP Compound.

Figure 2 shows a schematic of the system: the receiver monitors the feedback variables like round trip time $R \hat{T} T(t)$, loss ratio $\hat{l}(t)$, (as it is shown in the technical info tooltip of Skype) and periodically sends these information back to the sender by piggybacking them on data packets. The sender receives feedback data and adjusts the sending rate $r_{s}(t)$ accordingly by throttling both packet size and packet sending rate.

In order to derive how the feedback signal affects the Skype sending rate we consider step-like inputs which is

\footnotetext{
${ }^{1}$ Skype DSP hijacker: http://195.38.3.142:6502/skype/
} 


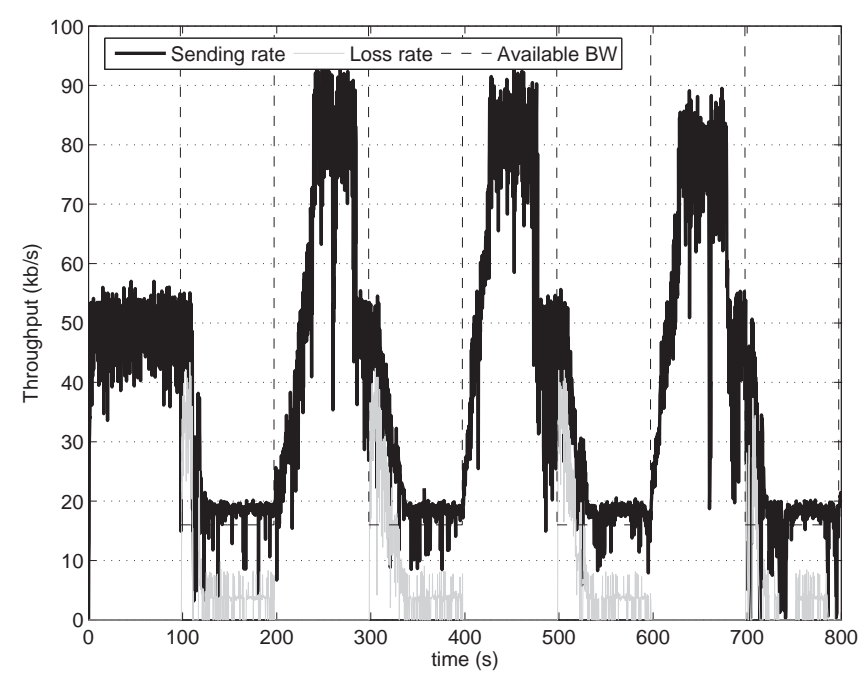

Fig. 3. Sending rate and loss rate in the presence of square wave available bandwidth of $200 \mathrm{~s}$ period

a basic practice when trying to investigate the dynamic behaviour of a system. In particular we will start by investigating Skype flows when in the presence of squarewave available bandwidths to figure out the effect of network available bandwidth on the Skype sending rate $r_{s}(t)$. Then we will study how variable link delay $R T T(t)$ and packet loss ratio $l(t)$ influence the sending rate $r_{s}(t)$. Finally, we will conjecture and verify a simple model for the Skype sending rate controller.

Before starting to report our results, it is worth noticing that Skype version we have used employs the adaptive codecs iSAC $^{2}$ and $\mathrm{iLBC}^{3}$ both developed by Global IP Sound.

\section{A. Skype dynamics over a square form wave available band- width}

This scenario aims at showing how the Skype sending rate reacts to sudden changes of available bandwidth. We employ an available bandwidth that varies as a square wave with maximum value $A_{M}=160 \mathrm{~kb} / \mathrm{s}$ and minimum value $A_{m}=16 \mathrm{~kb} / \mathrm{s}$ (see Figure 3 ).

We have run the experiment by setting the period of the square wave equal to $200 \mathrm{~s}$, which happened to be large enough to show all the transient dynamics.

Figure 3 shows that Skype decreases the sending rate when the link capacity drops from the value $A_{M}$ to the value $A_{m}$. The Skype flow takes approximately $40 \mathrm{~s}$ to track the available bandwidth during which it experiences a significant loss rate. It can be viewed that, when the available bandwidth drops, the loss rate increases to a peak value of $35 \mathrm{~kb} / \mathrm{s}$ whereas the sending rate reduces to less than $20 \mathrm{~kb} / \mathrm{s}$ in around $40 \mathrm{~s}$. By observing this behaviour it is very reasonable to conjecture that Skype implements a form of congestion control algorithm that reduces the sending rate when a high packet loss rate is measured. We will return soon on this

\footnotetext{
${ }^{2}$ http://www.gipscorp.com/files/english/datasheets/iSAC.pdf ${ }^{3}$ http://www.gipscorp.com/files/english/datasheets/iLBC.pdf
}
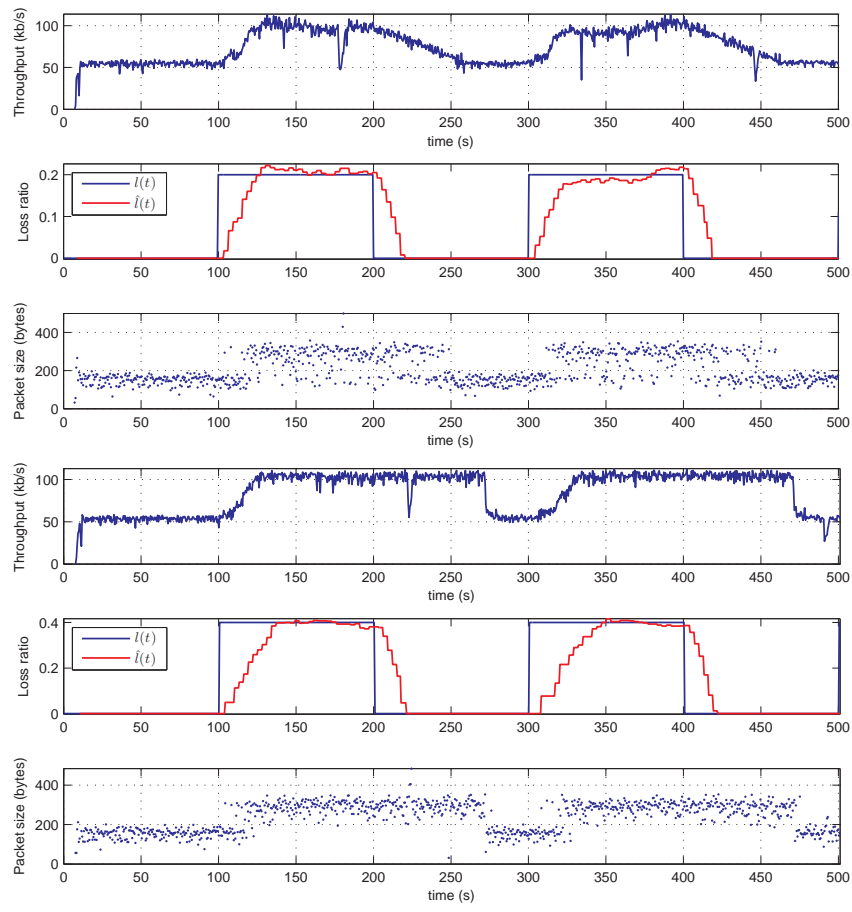

Fig. 4. Sending rate, loss ratio and packet size in the case of a square wave packet loss ratio with (a) $l_{M}=0.2$ and (b) $l_{M}=0.4$

feature by investigating how Skype behaves in the presence of packet loss rates.

\section{B. Skype over lossy link: throttling the loss ratio $l(t)$}

In the previous Section we have shown that Skype reacts to bandwidths shrinking by decreasing its sending rate. However, the experiment we have just described does not reveal the inputs of the congestion controller either the congestion control category to which Skype belongs, i.e. loss based, delay based or both. Towards this end, we now investigate the influence of packet losses on the Skype sending rate.

In order perform the investigation, the emulator injects packet losses which vary as a square wave having a maximum value of $l_{M}$ and a minimum value of 0 . The RTT is set to $100 \mathrm{~ms}$. We have logged the loss ratios as it is reported by the Skype application "Technical Call Info" and we have reported them in the next figures. We have considered a packet loss ratio which varies as a square-wave with period of $200 \mathrm{~s}$ and maximum values $l_{M}$ in the set $\{0.1,0.2,0.3,0.4,0.5\}$. Due to space constraints, we only report the most significant figures obtained for $l_{M}=0.2$ and $l_{M}=0.4$.

Figures 4(a) and (b) show two different behaviours of Skype in the presence of persistent losses on the link. In both cases Skype reacts to a persistent loss by increasing the transmission rate, i.e. the throughput. By looking at the packet size over time, in both cases we can see that when a persistent loss is detected the packet size is increased. This 

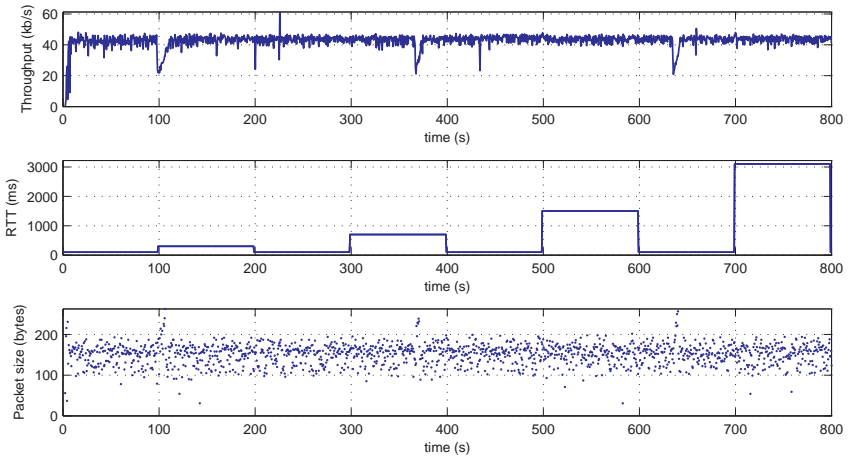

Fig. 5. Sending rate, loss ratio and packet size in the case of a variable link delay

fact suggests that Skype employs a Forward Error Correction (FEC) scheme in order to cope with persistent losses.

Figure 4 reveals other interesting facts: i) the Skype estimate of the loss ratio $\hat{l}(t)$ looks like a filtered version of the actual loss ratio $l(t)$ which has been set by using our emulation tool; ii) more interestingly, it seems that the signal $\hat{l}(t)$ drives the sending rate $r_{s}(t)$ in a roughly proportional way following as it can be argued by looking at $r_{s}(t)$ and $\hat{l}(t)$ dynamics; iii) in the case of $l_{M}=0.4$ (Figure $4(\mathrm{~b})$ ) the sending rate is kept at its maximum value of around $100 \mathrm{~kb} / \mathrm{s}$ even when $\hat{l}(t)$ reaches 0 . We argue that in this case Skype conservatively keeps on the FEC action for roughly $40 \mathrm{~s}$ because it measures a high value of losses. Other experiments have shown that such a behaviour is followed when $l_{M} \geq 0.3$, so that we can argue that Skype uses a threshold of $l_{M}$ between 0.2 and 0.3 to switch from one behaviour to the other.

Finally, it can be said that, due to the fact that the sending rate is not decreased when packet losses are detected, Skype does not employ a loss-based congestion control scheme.

\section{Skype over variable delay link: throttling the round trip time $R T T(t)$}

In this Section we investigate the effect of the end-to-end delay on the Skype sending rate. To the purpose we vary the link delay as shown in Figure 5 and we set the packet loss ratio to 0 . The available bandwidth is made large enough in order not to generate congestion on the link. The figure clearly shows that even very large variations in the $R T T$ (the last variation is $3.0 s$ ) do not produce any effect on the Skype sending rate. Thus we can also exclude that the application implements a delay based congestion control algorithm.

We have performed experiments in which the loss ratio $l(t)$ and $R T T(t)$ vary as in-phase square waves with the same period $(T=200)$, but we do not report the figures due to space constraints. Interestingly, we have found that the FEC action is inhibited when the $R T T$ increases to its maximum value. Therefore, it can be concluded that the $R T T$ influences the behaviour of the FEC action when in the presence of lossy links.

\section{The Skype Congestion Control Model}

\section{A. Modelling the Skype congestion control}

In Section III-A we have already shown the behaviour of Skype in the presence of a variable link capacity and we have found that Skype is able to match the available bandwidth to some extent after a significant transient time. In this subsection we provide a mathematical model to describe the dynamic behaviour of Skype.

We make the hypothesis (confirmed by the large number of experiments we have run) that the audio codec employed by Skype is multi-rate so that the encoder can select among $N$ levels $L_{k}=\left\{L_{1}, L_{2}, \ldots, L_{N}\right\}$ with $L_{1}<L_{2}<\ldots<L_{N}$. Moreover, we assume that the Skype adaptive codec is able to select the most appropriate mode according to some metric which should be the analogous of Carrier to Interference ratio $(\mathrm{C} / \mathrm{I})$ in the case of Adaptive Multi-Rate Wide Band (AMRWB) encoder [14]. Let $i(t)$ denote the switching law and let $L_{i(t)}$ be the encoder level at time $t$. It is very reasonable to assume that the switching law $i(t)$ is implemented by using if-then-else clauses, thus being very difficult to be identified.

We make the hypothesis that the Skype control law of the sending rate in the case of congestion is ruled by the following equation:

$$
r_{s}(t)=(1-\hat{l}(t)) \cdot(1+f(t)) L_{i(t)}
$$

where $f(t) \in[0,1]$ models the FEC action, meaning that when $f(t)=0$ the FEC action is off and when $f(t)=1$ the FEC action is at maximum. Basically, we conjecture that the switching function $i(t)$ selects the layer $L_{i(t)}$ based on the network conditions (RTT, jitter, loss ratio) and the rate is shrinked as much as the filtered loss ratio $\hat{l}(t)$ suggests. It is worth noting that (1) is able to explain the normal behaviour of Skype when no congestion or losses occur i.e. when $f(t)=0$ and $\hat{l}(t)=0$ when the sending rate results $r_{s}(t)=L_{i(t)}$ (see Figure 5).

We have run a set of experiments using a square wave available bandwidth with maximum value of $160 \mathrm{~kb} / \mathrm{s}$ and minimum value of $16 \mathrm{~kb} / \mathrm{s}$ with a period of $400 \mathrm{~s}$. We have shown the obtained results in Figure 6. In order to verify Eq. (1), we have conjectured the FEC action $f(t)$ shown in Figure 6 and we have supposed that $L_{i(t)}$ is set to $54 \mathrm{~kb} / \mathrm{s}$ during the experiment. Figure 6 shows both the Skype measured sending rate and predicted sending rate using (1). It can be seen that the model nicely predicts the Skype sending rate.

Now let us focus on the conjectured behaviour of $f(t)$ shown in Figure 6: the FEC is kept off until the first bandwidth increase happens at $t=400 \mathrm{~s}$. We conjecture that Skype infers a bandwidth increase when the RTT of the connection suddenly decreases and it triggers a "probing phase". Moreover, we think that Skype increases the FEC value when probing for bandwidth since losses can occur during this phase. Figure 6 shows that the FEC action is reduced to 0.2 when $\hat{l}(t)$ reaches 0 , and is then turned off later when $\hat{l}(t)$ is detected not to grow. 

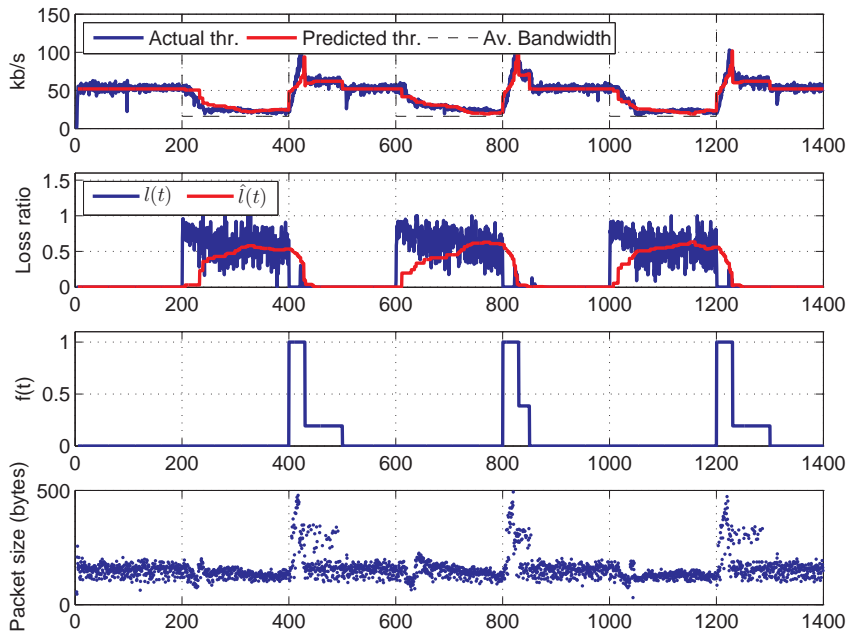

Fig. 6. Comparison between the actual and predicted rate using Eq. (1)

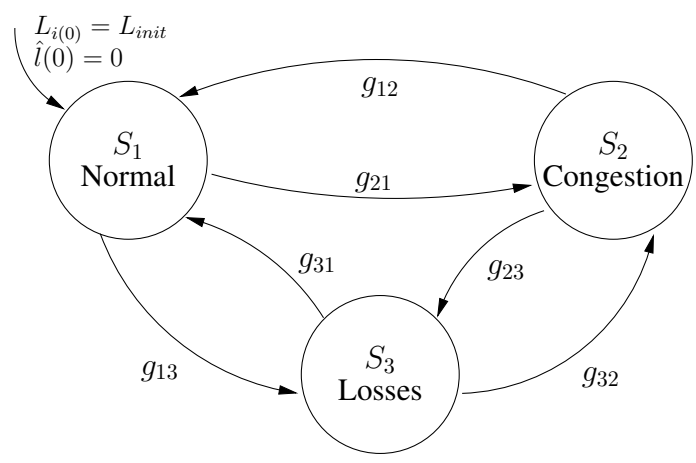

Fig. 7. Skype VoIP hybrid system model

It is worth to notice that the Equation (1) allows us to explain the long transient time that we have reported in Section III-A. The main driver of the Skype sending rate is in fact $\hat{l}(t)$, which is a low-pass filtered version of the actual loss ratio $l(t)$. The long transient exhibited by $\hat{l}(t)$ (see Figure 4) affects the sending rate response to congestion which is somewhat slow.

\section{B. The Skype Hybrid Automaton}

Building upon the results obtained by the investigations presented in Section III, we model the Skype VoIP sending rate using the hybrid automaton shown in Figure 7. In particular, the sending rate can exhibit three different dynamics depending on the state $S_{i}(i=1,2,3)$ of the automaton: in the state $S_{1}$, which is characterized by normal network conditions, i.e. no congestion occurs and no losses are present, the sending rate is kept unchanged; in the state $S_{2}$, which is triggered when Skype realizes that the network capacity has changed and congestion occurs, the sending rate is throttled using (1); in the state $S_{3}$, which is triggered when losses are present but are not due to network congestion (i.e. due to a lossy link, see Section III-B) the FEC action $f(t)$ is used in order to counteract the losses which Skype attributes to a lossy link.

\section{Hybrid modelling of a Skype Flow Accessing a Bottleneck}

In this Section we propose a hybrid automaton to model a Skype flow accessing a single bottleneck link characterized by an available bandwidth $b(t)$, a drop tail queue whose maximum size is $q_{M}$ and a round trip time $T$ which is the sum of the delay of the forward path $T_{1}$ and the delay of the backward path $T_{2}$. In the following we will denote with $x_{T}$ the signal $x$ delayed $T$ seconds and we will omit the time dependence of the signals for brevity. The evolution of the queue can be modelled by the following differential equation [5]:

$$
\dot{q}= \begin{cases}0 & q=0, r \leq b \text { or } q=q_{M}, r \geq b \\ r-b & \text { otherwise }\end{cases}
$$

where $r$ is the queue input rate. The queue overflow rate $o$ can be modelled as follows:

$$
o= \begin{cases}r-b & q=q_{M}, r>b \\ 0 & \text { otherwise }\end{cases}
$$

which means that when the queue is full the exceeding input rate $r-b$ is dropped [15].

Let us now consider the model of the sending rate of a Skype VoIP flow: equation (1) means that the Skype sending rate $r_{s}$ is mainly driven by the signal $\hat{l}$ which is a filtered version of the actual packet loss ratio $l=o / r$ measured at the sender after the delay $T_{2}$. Based upon experiments we assume that Skype filters $l_{T_{2}}$ using a first order low pass filter with a time constant $\tau$ :

$$
\dot{\hat{l}}=-\frac{1}{\tau} \hat{l}+\frac{1}{\tau} l_{T_{2}}
$$

which by considering that $l=o / r$ turns out:

$$
\dot{\hat{l}}=-\frac{1}{\tau} \hat{l}+\frac{1}{\tau} \frac{o_{T_{2}}}{r_{T_{2}}}
$$

By substituting (1) and (3) in (4), after straightforward computations we obtain:

$\dot{\hat{l}}= \begin{cases}f_{1}=\frac{1}{\tau}-\frac{\hat{l}}{\tau}-\frac{b_{T_{2}}}{\tau\left(1-\hat{l}_{T_{2}}\right)\left(1+f_{T_{2}}\right) L_{T_{2}}} & q=q_{M}, r>b \\ f_{2}=-\frac{1}{\tau} \hat{l} & \text { otherwise }\end{cases}$

Let $x=\left[\begin{array}{ll}\hat{l} & q\end{array}\right]^{T}$ denote the state of the system. It is simple to show that the state dynamics of the considered system can be described by means of the three state hybrid automaton $\mathcal{H}$ which is shown in Figure 8. In particular, the state $\Sigma_{1}$ holds when the queue is empty and the input rate is below the link available bandwidth, the dynamics of state is described by $\Sigma_{2}$ when the queue is neither full nor empty; the state $\Sigma_{3}$ describes the evolution of the system when the queue is full and the input rate is larger than the available bandwidth.

Lemma 1: The system $\Sigma_{3}$ has a unique equilibrium point:

$$
\hat{l}^{*}=1-\sqrt{\frac{b^{*}}{L^{*}\left(1+f^{*}\right)}} ; q^{*}=q_{M}
$$

when $b^{*}<\left(1+f^{*}\right) L^{*}$. Considered the system with no delay the equilibrium is asymptotically stable for perturbations $\Delta l \in[0,1]$. 


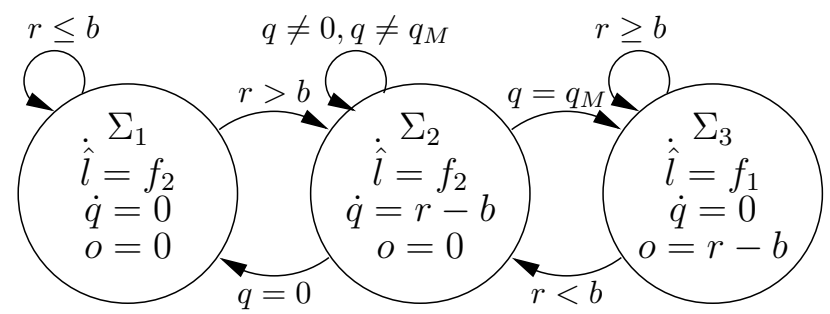

Fig. 8. The hybrid automaton model $\mathcal{H}$ of a Skype flow accessing a drop tail queue

Proof: Let us focus on the system $\Sigma_{3}$ which holds when the queue is full, i.e. when congestion occurs. By imposing $\dot{\hat{l}}=0$ and after straightforward computations we obtain (6). It is worth to notice that $\hat{l}^{*} \in[0,1]$ since the inequality $b^{*}<$ $\left.L^{*}\left(1+f^{*}\right)\right)$ holds. The proof is completed by employing the direct Lyapunov method using a candidate function $V(\hat{l})=$ $1 / 2\left(\hat{l}-\hat{l}^{*}\right)^{2}$ and by noticing that $\dot{V}(\hat{l})$ is definite negative.

Lemma 2: The system $\Sigma_{1}$ has a unique equilibrium point:

$$
\hat{l}^{*}=0 ; q^{*}=0
$$

when $b^{*}>\left(1+f^{*}\right) L^{*}$ which is globally asymptotically stable.

Proof: The lemma is proved by observing that $\Sigma_{1}$ is a linear system with one eigenvalue that is always strictly negative.

Lemma 3: The hybrid automaton $\mathcal{H}$ has a sink state $\Sigma_{3}$ if $b^{*}<\left(1+f^{*}\right) L^{*}$ or a sink state $\Sigma_{1}$ if $b^{*}>\left(1+f^{*}\right) L^{*}$.

Proof: The proof is omitted due to space limitation and it is contained in [16].

Proposition 1: By considering the equilibrium inputs $b^{*}$, $L^{*}$ and $f^{*}$ the hybrid automaton shown in Figure 8 has the following equilibrium state:

$$
\hat{l}^{*}=1-\sqrt{\frac{b^{*}}{L^{*}\left(1+f^{*}\right)}} ; q^{*}=q_{M}
$$

if $b^{*}<\left(1+f^{*}\right) L^{*}$ and:

$$
\hat{l}^{*}=0 ; q^{*}=0
$$

otherwise. Considered the system delay free system $\left(T_{2}=0\right)$ both the equilibria are asymptotically stable.

Proof: From Lemma 3 we know that if $b^{*}<\left(1+f^{*}\right) L^{*}$ then $\Sigma_{3}$ is a sink, so that for any initial condition $\left(\hat{l}_{0}, q_{0}\right) \in$ $X$ the state must end in $\Sigma_{3}$. Moreover, in Lemma 1 we proved that $\Sigma_{3}$ is an asymptotic stable equilibrium (8), so that we can conclude that for any $\left(\hat{l}_{0}, q_{0}\right) \in X$ the state will asymptotically converge to (8).

If we assume $b^{*}>\left(1+f^{*}\right) L^{*}$ following similar arguments we can conclude that for any any $\left(\hat{l}_{0}, q_{0}\right) \in X$ the state will asymptotically converge to (9).

Proposition 2: The controller employed by Skype is not able to counteract congestion episodes unless $L^{*}\left(1+f^{*}\right)<$ $b^{*}$.

Proof: Since we are under the hypothesis of Proposition 1 , we know that (8) is an asymptotically stable equilibrium for $\mathcal{H}$. Therefore by considering (3) and (8) we obtain a steady state value of the overflow rate given by:

$$
o^{*}=\sqrt{b^{*} L^{*}\left(1+f^{*}\right)}-b^{*}
$$

that is greater than zero if $b^{*}<L^{*}\left(1+f^{*}\right)$. In other terms, under congestion, the evolution of the system will be described by $\Sigma_{3}$ (Lemma 3 ) and thus the queue will be full $\left(q^{*}=q_{M}\right)$ and the overflow rate will be persistent $\left(o^{*}>0\right)$.

Remark 1: The steady state value of the loss rate $o^{*}$ does not depend on the time constant $\tau$ of the filter.

\section{CONCLUSIONS AND FURTHER WORK}

In this paper we propose a dynamic switching model of the congestion control algorithm implemented by the Skype VoIP application. By setting up an experimental testbed we have conjectured and verified what are the inputs and how they affect the Skype sending behaviour. Main findings are: (1) Skype does not implement a delay based control; (2) the sending rate matches the available bandwidth with a finite error, so that Skype is not able to avoid congestion properly; (3) the loss ratio is the main driver of the input rate dynamics.

As a further work we plan to relax the assumption $T_{2}=0$ in the proof of Proposition 1.

\section{REFERENCES}

[1] V. Jacobson, "Congestion avoidance and control," ACM SIGCOMM Computer Communication Review, 1988.

[2] R. Srikant, The Mathematics of Internet Congestion Control. Birkhäuser, 2004.

[3] S. Low, F. Paganini, and J. Doyle, "Internet congestion control," Control Systems Magazine, IEEE, vol. 22, no. 1, pp. 28-43, 2002.

[4] J. Lee, S. Bohacek, a. P. H. Jo and K. Obraczka, "Modeling communication networks with hybrid systems," IEEE/ACM Trans. Netw., vol. 15, no. 3, pp. 630-643, 2007.

[5] S. Mascolo, "Congestion control in high-speed communication networks using the Smith principle," Special Issue on "Control methods for communication networks” Automatica, vol. 35, no. 12, pp. 1921$1935,1999$.

[6] C. Hollot, V. Misra, and D. Towsley, "A control theoretic analysis of RED," IEEE INFOCOM '01, vol. 3, Apr. 2001.

[7] E. Kohler, M. Handley, and S. Floyd, "Designing DCCP: congestion control without reliability," ACM SIGCOMM '06, Sep. 2006.

[8] J. P. M. Handley, S. Floyd, "TCP Friendly Rate Control (TFRC): Protocol Specification," RFC 3448, Proposed Standard, Jan. 2003.

[9] P. Biondi and F. Desclaux, "Silver Needle in the Skype," BlackHat Europe '06, Mar. 2006.

[10] S. Baset and H. Schulzrinne, "An Analysis of the Skype Peer-to-Peer Internet Telephony Protocol,' IEEE INFOCOM '06, Apr. 2006.

[11] L. De Cicco, S. Mascolo, and V. Palmisano, "An Experimental Investigation of the Congestion Control Used by Skype VoIP," WWIC '07, vol. 4517, pp. 153-164, May 2007.

[12] - "Skype Video Responsiveness to Bandwidth Variations," in Proc. ACM NOSSDAV 2008, Braunschweig, Germany, May 28-30, 2008.

[13] L. Rizzo, "Dummynet: a simple approach to the evaluation of network protocols," ACM SIGCOMM Computer Communication Review, vol. 27, no. 1, pp. 31-41, 1997.

[14] B. Bessette et al, "The adaptive multirate wideband speech codec (AMR-WB)," Speech and Audio Processing, IEEE Transactions on, vol. 10, no. 8, pp. 620-636, 2002.

[15] S. Mascolo, "Modeling the Internet congestion control using a Smith controller with input shaping," Control Engineering Practice, vol. 14, no. 4, pp. 425-435, Apr. 2006.

[16] L. De Cicco, S. Mascolo, and V. Palmisano, "A Mathematical Model of the Skype VoIP Congestion Control Algorithm," tech. report TR-08-07, Politecnico di Bari, 2008. [Online]. Available: http://c3lab.poliba.it/downloads/skype_tr0807.pdf 